\title{
RENORMALIZED EFFECTIVE HAMILTONIAN APPROACH TO QCD
}

\author{
D. G. Robertson, ${ }^{1}$ E. S. Swanson, ${ }^{1}$ A. P. Szczepaniak, ${ }^{2}$ C.-R. Ji ${ }^{1}$ and S. R. Cotanch ${ }^{1}$ \\ ${ }^{1}$ Department of Physics, North Carolina State University, Raleigh, NC 27695, USA \\ ${ }^{2}$ Department of Physics and Nuclear Theory Center, Indiana University, \\ Bloomington, IN 47405, USA
}

\begin{abstract}
Continuing our previous QCD Hamiltonian studies in the gluonic and quark sectors, we describe a new renormalization procedure which generates an effective Hamiltonian. The formulation, which is in the Coulomb gauge, provides an improved framework for investigating hadron structure.
\end{abstract}

\section{Introduction}

For the past few years the NCSU group has focused upon the development of a comprehensive relativistic, many-body description of hadron structure1 1 . This approach has many virtues, particularly the explicit incorporation of gluonic degrees of freedom and a rigorous connection to QCD at short distances. Calculations of the glueball spectrum have been quite encouraging, reproducing the available lattice data.

This talk details recent results from a new renormalization procedure for the Coulomb gauge QCD Hamiltonian. There are several reasons for selecting the Coulomb gauge. First, it is completely physical; there are no spurious gauge degrees of freedom or ghosts. Second, both $q \bar{q}$ and gluon-gluon interactions appear explicitly in the canonical Hamiltonian which facilitates contact with quark model phenomenology. Finally, there appears to be a natural scenario for confinement in the Coulomb gauge, as originally suggested by Gribovi and recently formalized by Zwanzigert.

This Hamiltonian must be supplemented with a regulator, which is applicable nonperturbatively and is consistent with the many-body techniques we eventually wish to employ. Next the renormalized Hamiltonian is constructed, which includes the correct cut-off dependent counterterms such that physical quantities (e.g., eigenvalues) are cut-off independent. It is inevitable that the regulators at our disposal will violate subsets of Lorentz covariance and gauge invariance, so that the structure of the counterterm Hamiltonian may be expected to be quite complicated. Due to asymptotic freedom, however, it should be reasonable to construct these operators using perturbation theory, provided the energy cut-off is not too low.

The result is a Hamiltonian formulation of QCD which is fully covariant and gauge invariant through some order in perturbation theory. To effectively apply this Hamiltonian, further approximations and phenomenological considerations are necessary. An 
approach we have initiated involves making a variational Ansatz for the physical vacuum state, based on the BCS "pairing" form. Quasi-particle degrees of freedom emerge naturally with constituent-scale masses and may be identified with constituent quarks and gluons. One then can construct approximate bound states of these constituents utilizing standard many-body techniques such as Tamm-Dancoff and RPA.

\section{Regularization and Renormalization}

Our starting point is the canonical QCD Hamiltonian in the Coulomb gauge $\nabla \cdot \mathbf{A}=0$. The dynamical degrees of freedom are the transverse gauge fields $\mathbf{A}$, their conjugate momenta $\boldsymbol{\Pi}$, and the quark field $\psi$. The canonical Hamiltonian may be written as

$$
H_{\text {can }}=H_{0}+H_{g g}+H_{q g}+H_{C},
$$

where $H_{0}$ is the free Hamiltonian for quarks and transverse gluons, $H_{g g}$ contains the self-interactions between transverse gluons, and $H_{q g}$ contains quark-gluon interactions. $H_{C}$ is the Coulomb term,

$$
H_{C}=\frac{1}{2} g^{2} \int d^{3} x d^{3} y \mathcal{J}^{-1} \rho^{a}(\mathbf{x}) K^{a b}(\mathbf{x}, \mathbf{y}) \mathcal{J} \rho^{b}(\mathbf{y}),
$$

where $\mathcal{J}=\operatorname{det}[\nabla \cdot \mathbf{D}]$ is the Fadeev-Popov determinant, and the kernel $K$ is given by

$$
K^{a b}(\mathbf{x}, \mathbf{y})=\left\langle\mathbf{x}, a\left|(\nabla \cdot \mathbf{D})^{-1}\left(-\nabla^{2}\right)(\nabla \cdot \mathbf{D})^{-1}\right| \mathbf{y}, b\right\rangle
$$

The color charge density $\rho^{a}$ includes both matter and gluonic contributions, $\rho^{a}(\mathbf{x})=$ $\psi^{\dagger}(\mathbf{x}) T^{a} \psi(\mathbf{x})+f^{a b c} \mathbf{A}^{b}(\mathbf{x}) \cdot \boldsymbol{\Pi}^{c}(\mathbf{x})$, so that $H_{C}$ represents a complicated set of instantaneous quark-quark, quark-gluon, and gluon-gluon interactions.

Perturbative schemes for renormalizing Hamiltonians typically suffer from technical problems related to the occurrence of vanishing energy denominators. One way to avoiding this difficulty is the cut-off method of Głazek and Wilson $\mathbf{3}$. In this approach one considers the basis formed by eigenstates of the free Hamiltonian $H_{0}$, with eigenvalues $E_{n}$. The theory is then regulated by suppressing matrix elements of $H$ between states for which the absolute value of the free energy difference $E_{n m} \equiv E_{n}-E_{m}$ is large. Specifically, we define matrix elements of the regulated Hamiltonian $H(\Lambda)$ by

$$
\langle n|H(\Lambda)| m\rangle \equiv E_{n} \delta_{n m}+f_{m n}(\Lambda)\left\langle n\left|H_{I}\right| m\right\rangle,
$$

where $H_{I}=H-H_{0}$ is the interaction. Here $f_{n m}(\Lambda)$ is some convenient function which is unity for $\left|E_{n m}\right| \ll \Lambda$ and vanishes for $\left|E_{n m}\right| \gg \Lambda$. In Ref. 1 we chose

$$
f_{n m}(\Lambda)=e^{-E_{n m}^{2} / \Lambda^{2}}
$$

Note that, since the cut-off is defined in terms of free energy differences, it is not fully Lorentz covariant (though it is of course rotationally invariant). It also violates gauge invariance, though this issue is difficult to address directly in the present context since we work in a fixed gauge. The counterterm Hamiltonian will therefore also contain Lorentz- and gauge-noninvariant terms, which are necessary to correct for the violations 
induced by the regulator. These complications are in practice unavoidable if one wishes to work with Hamiltonians in a relativistic field theory.

At this point we have a fully regulated formulation of QCD in the Coulomb gauge. Our next task is to remove the dependence on the cut-off parameter $\Lambda$ by adding counterterms to the Hamiltonian. We thus obtain the renormalized effective Hamiltonian, $H_{\text {eff }}(\Lambda)$, which may then be analyzed nonperturbatively using many-body techniques.

An elegant way of determining the counterterms involves designing a similarity transformation which changes the cut-off in all matrix elements of $H$. Because the transformed Hamiltonian is equivalent to the original one, physical predictions (e.g., eigenvalues) are unchanged. One then searches for Hamiltonians which are form invariant, or "coherent," under this transformation. Such self-similar Hamiltonians will automatically yield cut-off independent predictions and thus represent renormalized Hamiltonians. Specifically, we write

$$
H\left(\Lambda^{\prime}\right)=U\left(\Lambda^{\prime} ; \Lambda\right) H(\Lambda) U^{\dagger}\left(\Lambda^{\prime} ; \Lambda\right)
$$

where $U$ is a unitary matrix constructed so that the matrix elements $\left\langle m\left|H\left(\Lambda^{\prime}\right)\right| n\right\rangle$ are all proportional to $\exp \left(-E_{n m}^{2} / \Lambda^{\prime 2}\right)$. Copstruction of a suitable $U$ is may be accomplished following the formulation of Wegner 6 . We define the operator $T$ via

$$
\frac{d U\left(\Lambda ; \Lambda^{\prime}\right)}{d\left(\Lambda^{-2}\right)} \equiv T(\Lambda) U\left(\Lambda ; \Lambda^{\prime}\right)
$$

The evolution of $H$ under a change in $\Lambda$ is then given by

$$
\frac{d H(\Lambda)}{d \Lambda^{-2}}=[T(\Lambda), H(\Lambda)]
$$

from which $T$ is seen to be a generator of infinitesimal scale transformations. It is straightforward to show that the choice

$$
T(\Lambda)=\left[H_{0}, H(\Lambda)\right]
$$

results in the transformation properly changing the value of the cut-off parameter in the regulating functions used for all matrix elements of the Hamiltoniant 1 .

Eqn. (8) can then be analyzed order-by-order in perturbation theory by expanding the interaction $H_{I}=\sum_{p} g^{p} H_{p}$. At second order, for example, we find

$$
\left\langle n\left|\delta V_{2}\right| m\right\rangle=\frac{1}{2} \sum_{l}\left(\frac{1}{E_{n l}}+\frac{1}{E_{m l}}\right)\left(e^{-2 E_{n l} E_{m l} / \Lambda^{2}}-e^{-2 E_{n l} E_{m l} / \Lambda^{\prime 2}}\right)\left\langle n\left|V_{1}(\Lambda)\right| l\right\rangle\left\langle l\left|V_{1}(\Lambda)\right| m\right\rangle,
$$

where $\left\langle n\left|H_{p}(\Lambda)\right| m\right\rangle \equiv \exp \left(-E_{n m}^{2} / \Lambda^{2}\right)\left\langle n\left|V_{p}(\Lambda)\right| m\right\rangle$ defines the "reduced" interaction $V_{p}$, and $\left\langle n\left|\delta V_{2}\right| m\right\rangle \equiv\left\langle n\left|V_{2}\left(\Lambda^{\prime}\right)\right| m\right\rangle-\left\langle n\left|V_{2}(\Lambda)\right| m\right\rangle$. This shift in the interaction explicitly accounts for the physics removed by changing the cut-off from $\Lambda$ to $\Lambda^{\prime}$.

As discussed above, requiring form-invariance of the Hamiltonian under the similarity transformation determines the counterterm structure uniquely. We have implemented this constraint and determined the complete effective Hamiltonian through 
second order. The operators that appear in the counterterm Hamiltonian include, as expected, all possible one-body operators which are rotationally invariant (and respect, e.g., time-reversal invariance, charge conjugation, etc.). These are a gluon "mass" term, $\mathbf{A}^{2}$, the operators $\boldsymbol{\Pi}^{2}$ and $\left(\nabla_{i} A_{j}\right)^{2}$, and, for fermions, $\bar{\psi} \psi$. Each has a calculable coefficient, except for the particular combination of $\boldsymbol{\Pi}^{2}$ and $\left(\nabla_{i} A_{j}\right)^{2}$ which has the structure of a gluon wavefunction renormalization. For this combined operator, the variation of the coefficient under changes in the cut-off is calculable, and gives the gauge field anomalous dimension: $\gamma_{A}=-\left(g^{2} / 16 \pi^{2}\right)\left(4 C_{A} / 3\right)$, where $C_{A}$ is the Casimir invariant of the adjoint representation. See Ref.t for full details.

\section{Work in Progress and Future Directions}

Having obtained the renormalized Hamiltonian, we can now proceed to construct a model for the ground state and analyze excitations such as glueballs, mesons and hybrids. In Ref. 1 , we focused attention on the gluonic sector and made a variational BCS vacuum Ansatz. This leads to a gap equation which may be solved numerically to give the spectral function of the quasi-particle constituent gluons.

Work in progress includes extending the similarity analysis to higher orders, applying this smooth cut-off treatment to the quark sector and designing improved models for the vacuum. At third order, one should see the running of the coupling, as well as the appearance of many new operators in the counterterm Hamiltonian. Going beyond BCS is expected to be particularly important in a combined treatment of the quark and gluon sectors. In addition, other composite operators, such as currents, must be properly renormalized which is also possible using the formalism presented in Ref.t.

Finally, there are a host of phenomenological applications to be explored. Not only meson spectra but also form factors, electro-weak and hadronic decays, etc. can be predicted and confronted with available data. This comprehensive analysis should provide significant insight into hadron structure as well as a key linkage between fundamental QCD and the successful phenomenological quark model.

\section{Acknowledgments}

Financial support is provided by U. S. Department of Energy.

\section{References}

1. D. G. Robertson, E. S. Swanson, A. P. Szczepaniak, C.-R. Ji, and S. R. Cotanch, submitted to Phys. Rev. D.

2. A. Szczepaniak, E. S. Swanson, C.-R. Ji, and S. R. Cotanch, Phys. Rev. Lett. 76, 2011 (1996); A. Szczepaniak and E. S. Swanson, Phys. Rev. D 55, 1578 (1997); E. S. Swanson and A. Szczepaniak, hep-ph/9804219.

3. V. N. Gribov, Nucl. Phys. B 139, 1 (1978).

4. D. Zwanziger, Nucl. Phys. B 485, 185 (1997); A. Cucchieri and D. Zwanziger, Phys. Rev. Lett. 78, 3814 (1997).

5. St. D. Głazek and K. G. Wilson, Phys. Rev. D 48, 5863 (1993); ibid. 49, 4214 (1994).

6. F. Wegner, Ann. Physik 3, 77 (1994). 